\title{
ERDRÜCKENDE NACHBARSCHAFT MEXIKOS BEZIEHUNGEN ZU DEN USA
}

\author{
Von Wolf GrabendorfF
}

Kein Geringerer als Präsident Carter zitierte jüngst in einer Tischrede anläßlich des Besuchs des mexikanischen Präsidenten López Portillo den Porfirio Diaz zugeschriebenen Satz: „Armes Mexiko - so fern von Gott und so nahe den Vereinigten Staaten"1! Dieser Ausspruch ist immer noch die kürzeste Formel, um die Beziehungen zwischen der Weltmacht USA und seinem armen Nachbarn Mexiko zu beschreiben. Es handelt sich um eine Nachbarschaft besonderer Art, die wenig gemein hat mit der Nachbarschaft, die die USA im Norden mit Kanada verbindet ${ }^{2}$. Denn im Süden grenzen die USA an die Dritte Welt, und der Rio Grande ist mehr Kulturschwelle als gewöhnliche Grenze. Die Probleme und Konflikte, die sich zwischen Mexiko und den USA ergeben, sind daher nicht nur Nachbarschaftsprobleme, sondern auch Gefälleprobleme. Sie sind besonders schwierig immer dann zu lösen, wenn sich Nachbarschaftsprobleme mit Gefälleproblemen vermischen und so die Empfindlichkeiten auf beiden Seiten potenzieren. Diese besondere Art der Beziehungen, die sich zwischen Mexiko und den USA entwickelt haben, haben dem kleineren und schwächeren Land die Notwendigkeit aufgezwungen, spezifische Methoden und Instrumente zu entwickeln, um mit dem erdrückenden Nachbarn leben zu können. Es soll an dieser Stelle bewußt nicht so sehr von den Schwierigkeiten, die die USA mit Mexiko haben, die Rede sein, sondern von der Problematik dieser Beziehungen aus mexikanischer Sicht ${ }^{3}$.

Es spricht vieles dafür, daß Mexiko den Nord-Süd-Konflikt gegenüber den USA seit langem vorexerziert hat, und daß viele Lehren, die die USA für ihre Lateinamerika-Politik und auch für ihre Politik gegenüber der Dritten Welt insgesamt gezogen haben, dieser konflikt- und spannungsreichen Beziehung zu Mexiko entspringen ${ }^{4}$. Für die USA bedeutet das Verhältnis zu Mexiko gleichzeitig die erste

1 Vgl. "NZZ ${ }^{\alpha}$ vom 18. 2. 1977.

2 Das schließt nicht aus, daß es vor allem in den letzten Jahren zur Entdeckung gemeinsamer Probleme zwischen Kanada und Mexiko gekommen ist. Vgl. die ausführliche Darstellung bei Farrell, Barry F. (Hrsg.): América Latina y Canada frente a la política exterior de los Estados Unidos, México 1975.

3 Die weitaus ergiebigsten Analysen sind von den Mitarbeitern des "Colegio de México" erstellt worden. Neben den Beiträgen zu diesem Thema in der Zeitschrift Foro Internacional, sind vor allem folgende Titel benutzt worden: Ojeda, Mario: Alcances y límites de la política exterior de México, México 1976. Centro de Estudios Internacionales (Hrsg.): México y América Latina: La nueva política exterior, México 1974. Centro de Estudios de México (Hrsg.): La política exterior de México: Realidad y perspectivas, Mexico 1972. Aus nordamerikanischer Sicht ist die Analyse von Schmitt, Karl M.: Mexico and the United States, 1821-1973: Conflict and Coexistence, New York u. a. 1974, bei weitem die beste. Für eine aktuelle und sehr weitsichtige Bestandsaufnahme der Beziehungen aus US-Sicht vgl. Fagan, Richard R.: "The Realities of U.S.-Mexican Relations*, in: Foreign Affairs, Vol. 55, Nr. 4 (Juli 1977), S. $685-700$.

4 Die Beziehungen der Vereinigten Staaten zu Mexiko im Laufe der Geschichte, erscheinen in einem besonderen Licht, wenn man bedenkt, daß im wesentlichen dieselbe Politik auf Lateinamerika im allgemeinen und später auf die übrige Welt übertragen wurde. Die offizielle Doktrin der amerikanischen Außenpolitik in neuerer Zeit, wurde zum großen Teil auf dem "mexikanischen Amboß zurechtgeschmiedet. So Frank Tannenbaum in seinem Buch, Mexiko - Gesicht eines Landes, Stuttgart 1967, S. 229 (Originalausgabe: Mexico, the Struggle for Peace and Bread, New York 1951). Vgl. dazu außerdem Arnold Toynbeen's prophetische Worte zur US-Außenpolitik, die die Rolle Mexicos für den Lernprozeß der USA sehr gut kennzeichnen: „The states of Europe and Asia within the United States' sphere of influence, are going to be as touchy as the Latin American States, and the United States is likely to handle them by diplomatic techniques, that she has learnt from her Latin American experience.* Zitiert bei Cline, Howard F.: The United States and Mexico, New York 1965, S. 6. 
Auseinandersetzung mit einer Revolution in einem Land der Dritten Welt. Die späteren Konflikte mit Cuba, Vietnam und schließlich China - um nur einige zu nennen - waren zumindest teilweise durch die historische Erfahrung mit Mexiko geprägt ${ }^{5}$.

\section{Kulturschwelle Rio Grande}

Ein ganzes Bündel von Faktoren läßt die über 3000 km lange Grenze zwischen den USA und Mexiko zur Kulturschwelle und zur Grenze zwischen Erster und Dritter Welt werden. Dazu gehört an erster Stelle die spezifische geographische Situation, die ebenso wie die historische Erfahrung von Revolution und Intervention die große Kluft zwischen Nord und Süd an dieser Stelle hat entstehen lassen. Ein weiteres Merkmal der Kulturschwelle ist die unterschiedliche ethnische und kulturelle Tradition auf beiden Seiten, die zwei sehr konträre politische Kulturen geprägt hat. Schließlich stehen sich auf der nördlichen Seite dieser Kulturschwelle Kapital und Technologie und auf ihrer südlichen Seite Arbeitskraft und Rohstoffe in einem nur schwer auszugleichenden Gegensatz gegenüber. Es ist also keine "normale" Grenze, die zwischen Mexiko und den USA verläuft, und je brisanter die Probleme auf beiden Seiten werden, desto deutlicher wird die Grenze nicht nur in der Auseinandersetzung zwischen beiden Ländern, sondern auch in ihrer physischen Erscheinungsform ${ }^{6}$.

Die geographische Lage hat eine sehr dynamische und in vieler Beziehung einzigartige Beziehung zwischen Mexiko und den USA entstehen lassen, wobei die Mexikaner lange geglaubt haben, daß ihnen ihre geographische Situation zum Vorteil gereiche, weil die Ströme von Menschen, Waren, Kapital und Technologie aus diesem Grunde einfacher und kostengünstiger zwischen den beiden Nachbarn hinund herfließen könnten. Die Entwicklung sollte jedoch zeigen, wie problematisch dieser Austausch zwischen Nord und Süd für die mexikanische Entwicklung werden sollte und als wie schwierig sich die Regulierung dieser Ströme an der Kulturschwelle des Rio Grande erwies.

Historisch gesehen, waren die Beziehungen von vier sehr unterschiedlichen Phasen geprägt? 1822 bis 1917 war Mexiko dem Expansionsdrang der USA fast pausenlos ausgesetzt. Es war ein knappes Jahrhundert der Interventionen und Grenzstreitigkeiten, in dem die junge Republik mehr Energien zur Abwehr aufbringen mußte, als sie zum Aufbau der eigenen Nation verwenden konnte. Allein zwischen 1835 und 1855 verlor Mexiko fast die Hälfte seines Territoriums - die heutigen USStaaten Texas, New Mexico, Arizona, Kalifornien, Nevada und Utah - an den expandierenden Nachbarn ${ }^{8}$. Von 1917 bis 1940 waren die Auseinandersetzungen

5 Vgl. dazu Beiträge in Higham, Robin (Hrsg.): Intervention or Abstention: The Dilemma of American Foreign Policy, Lexington 1975 .

6 Stellenweise ist die Grenze bereits durch meterhohe Drahtzäune, elektrische Kontrollmechanismen, Hubschrauber- und Hundepatrouillen gesichert, so daß von mexikanischer Seite der Vergleich mit der "Mauer" in Berlin nicht gescheut worden ist.

7 Vgl. auch die Aufstellung bei Weil, Thomas E. u. a.: Area Handbook for Mexico, Washington, 2. Aufl. 1975, S. 263.

8 Für eine kompetente Interpretation der US-Úbergriffe auf Mexico siehe: Cline, a. a. O., S. 7-16. Zum Scndungsbewußtsein der USA und seinen Auswirkungen auf Lateinamerika siehe auch Hirsch-Weber, Wolfgang: Lateinamerika: Abhängigkeit und Selbstbestimmung, Opladen 1972, S. 128-140; und die dort aufgeführten Beispiele und Literaturangaben. $\mathrm{Zu}$ den eigentlichen Interventionen vgl. die ausgezeichnete Arbeit von Haley, Edward P.: Revolution and Intervention. The Diplomacy of Taft and Wilson with Mexico 1910-1917, Cambridge/Mass. 1970. Sowie Quirk, Robert E.: An Affair of Honor, Woodrow Wilson and the Occupation of Veracruz, New York 1962. Sowie aus mexikanischer Sicht: García Cantú, Gastón: Las invasiones norteamericanas en México, Mexico 1971. 
mit den USA eine direkte Folge der Regelungen der mexikanischen Verfassung und ihrer Auswirkung auf US-Eigentum und -Interessen. In dieser Zeit galt es für die Mexikaner, die Souveränität über ein eigenes Wirtschafts- und Gesellschaftssystem zu erringen. Die dritte Phase von 1940 bis 1952 war die der engsten Zusammenarbeit mit den USA, geprägt durch den Krieg, die Nachkriegsereignisse und den beginnenden Kalten Krieg. Von 1952 bis 1970 ließ sich erstmals eine gewisse Entfremdung zwischen den USA und Mexiko aufgrund der Lateinamerika-Politik der USA erkennen, die sich aber nur in begrenzten Konflikten niederschlug und gleichzeitig durch engste wirtschaftliche Verflechtung kompensiert zu werden schien. Rückblickend sind die drei Jahrzehnte von 1940 bis 1970 nicht umsonst als die „era of good feeling“ bezeichnet worden. Mit dem Amtsantritt Echeverrías 1970, der zusammen mit Cárdenas zu den umstrittenen Präsidenten Mexikos gehört ${ }^{9}$, begann in Mexiko die offene Identifikation mit der Dritten Welt und der Versuch, den Einfluß und die Abhängigkeit von den USA abzubauen. Diese Entwicklung war sicherlich stärker durch die Systemkrise der mexikanischen Politik und die Stagnation der mexikanischen Wirtschaft hervorgerufen worden als durch direkten USEinfluß. Aus innenpolitischen Gründen wurde es geradezu notwendig, sich gegen das Entwicklungsmodell der USA abzugrenzen und nach neuen Wegen für Mexikos Probleme zu suchen ${ }^{\mathbf{1 0}}$.

Den USA konnte diese Entwicklung keineswegs gleichgültig bleiben, und deswegen war insbesondere das letzte Jahr der Echeverría-Administration mit seinen immer deutlicher zutage tretenden Wirtschaftsproblemen und den Peso-Abwertungen, gekennzeichnet von Gerüchten um eine Destabilisierung Mexikos durch die USA. Auf beiden Seiten wurden die Konflikte nicht mehr einzeln und pragmatisch gesehen, sondern nur noch in ihrem ideologischen Bezugsrahmen ${ }^{11}$. Dabei hat Echeverría im Gegensatz zu vielen Interpretationen keineswegs fundamentale Änderungen im außenpolitischen Verhalten gegenüber den USA vorgenommen. Vielmehr hat er die Bandbreite des mexikanischen Systems nur stärker genützt und stilistisch einige andere Akzente gesetzt als seine Vorgänger im $\mathrm{Amt}^{12}$. Für ihn waren die Außenbeziehungen eine Möglichkeit, durch zusätzliche „bargaining power" gegenüber den USA größeren Spielraum für die Neuordnung der stagnierenden Wirtschaft zu gewinnen ${ }^{13}$.

Die tiefgreifenden Auseinandersetzungen zwischen beiden Staaten haben dazu geführt, daß die USA trotz ihres starken Engagements den Mexikanern einen größeren Spielraum eingeräumt haben als vielen anderen lateinamerikanischen und insbesondere karibischen Ländern ${ }^{14}$. Den Mexikanern gelang es, sich mit den USA zu

9 Für eine vorzügliche Ưbersicht über die Regierungszeit Echevarrías, siehe Mols, Manfred: „Mexico unter Luis Echeverría Alvarez (1970-1976)“ in: Mols/Tobler: Mexico: Die institutionalisierte Revolution, $\mathrm{Köln} /$ Wien 1976, S. 171-233.

10 Der Verfasser hat an anderer Stelle versucht, die außerordentliche Bedeutung der Außenpolitik für die Stabilisierung des mexikanischen Systems darzustellen; siehe Grabendorff, Wolf: "Die Außenpolitik Mexicos und ihre innenpolitischen Ziele", in: Berichte zur Entwicklung in Spanien, Portugal und Lateinamerika (Nov./Dez. 1976), Heft 8, S. 40-48.

11 So äußerte ein hoher mexikanischer Beamter während des Kissinger-Besuchs 1976 in Mexiko, „the bilateral problems between us are secondary to the fact that an ideological confrontation seems to be forming" in: "N. Y. T." vom 14. 6. 1976.

12 Vgl. Poitras, "Guy E.: "Mexico's ,New“ Foreign Policy“, in: Inter-American Economic Affairs, Vol. 28, Nr. 3 (Winter 1974), S. 65/66.

13 So auch Brody, Olga de: "Mexico in the 1970 s and its Relations with the United States", in: Latin America and the United "States: The Changing Political Realities, hrsg. von Cotler, Julio/Fagen, Richard, Stanford 1974, S. 314-333, hier S. 315.

14 "The result has been probably less US-interference in Mexican affairs than in most other Caribbean countries." Vgl. Blasier, Cole: The Hovering Giant: US Responses to Revolutionary Change in Latin America. Pittsburgh 1976, S. 235. 
arrangieren, ohne die Ergebnisse ihrer Revolution von 1910-1917 grundsätzlich zu gefährden ${ }^{15}$. Gerade die Notwendigkeit, sich gegen die erdrückende Macht im Norden abzugrenzen, hat zur nationalen Identität und einer sehr bewußten, eigenen politischen Kultur ${ }^{16}$ beigetragen. Psychologisch gesehen ist der Antiamerikanismus nur selten zum echten Yankeehaß geworden. Vielmehr steht der Ablehnung des starken US-Einflusses durchaus die Bewunderung der Leistung der USA und die Hoffnung, einen gleichen Lebensstandard zu erreichen, gegenüber. Als Staat hat sich Mexiko gegenüber den USA zwar immer unabhängig, aber durchaus kooperativ verhalten. Im Gegenzug haben die USA kein Regime in Mexiko - einschließlich das des heftig kritisierten Präsidenten Echeverría - trotz weiter Bereiche mangelnder Übereinstimmung als feindlich betrachtet, wie das bei Castros Cuba und Allendes Chile der Fall war ${ }^{17}$.

Bis Ende der sechziger Jahre waren die Bedingungen der „special relationship“ von beiden Seiten als vorteilhaft akzeptiert worden. Die USA glaubten, aus der rapiden wirtschaftlichen Entwicklung Mexikos durch starken Kapital- und Technologietransfer Nutzen ziehen zu können, und die Mexikaner meinten, mit Billiglohnproduktion und Rohstoffexport, Wanderarbeit und Tourismus, den großen Markt der USA für die eigene Entwicklung nutzbar machen zu können ${ }^{18}$. Erst die Krise der siebziger Jahre hat die Gefahren dieser internationalen Arbeitsteilung deutlich werden lassen. Die Aufstellung bzw. das Ausbleiben von Steuerungsmechanismen für die importierten Arbeitskräfte und Rohstoffe in den USA und das importierte Kapital und die Technologie in Mexiko haben neue Konfliktstoffe geschaffen. Dabei hat der Versuch der jeweiligen Seite, zur Sicherung ihrer nationalen Interessen in das völlig andersartige System des Nachbarn hineinzuregieren, besonders starke Friktionen und Belastungen hervorgerufen. Dem schwächeren Partner Mexiko blieb dabei oft keine andere Wahl, als durch die Solidarisierung mit Ländern ähnlicher Problematik Druck auf die USA auszuüben. Andererseits versuchten die USA am Beispiel Mexiko klarzustellen, daß eine Veränderung des bisherigen Systems der Wirtschaftsbeziehungen zwischen Industrie- und Entwicklungsländern nur bis zu einem gewissen Grad hingenommen werden kann.

Die Beziehungen zwischen Mexiko und den USA machen in ihrer Beispielhaftigkeit für die Süd-Nord-Beziehung überhaupt eine Untersuchung der dabei entstehenden Probleme und ihrer Lösungsmöglichkeiten besonders interessant. Andererseits sind die spezifischen Eigenheiten der Grenze am Rio Grande so stark, daß bei der Analyse der Konflikte zwischen Mexiko und den USA sehr genau zwischen denen der eigentlichen Nachbarschaft und denen des Gefälles unterschieden werden muß.

\section{Konfliktraster zwischen Mexiko und den USA}

Seit dem Zweiten Weltkrieg haben Interaktionen jeder Form zwischen beiden Staaten außerordentlich zugenommen, so daß die Nachbarschaftsprobleme auf beiden Seiten der Kulturschwelle des Rio Grande immer deutlicher spürbar wurden. Der

15 Vgl. dazu Halay, a. a. O., S. 266. Die Strategie des US-Response hat vergleichend (Mexiko-BolivienCuba - Guatemala) Blasier, a. a. O., vorzüglich dargestellt. Siehe dort auch die Beschreibung der Detailkonflikte im Falle Mexikos, S. 101-128.

16 Zur politischen Kultur Mexikos vgl. Schelling, Cornelia v.: „Grundzüge der politischen Kultur Mexikos*. in: Berichte zur Entwicklung in Spanien, Portugal und Lateinamerika (Nov./Dez. 1976), Heft 8, S. 3-9.

17 Dazu: Fauriol, Georges: The Foreign Policy of Mexico, Washington 1974, unveröffentlichtes Manuskript, S. 49.

18 Vgl. Brody, a. a. O., S. 314. 
Strom von Menschen, Waren und Kommunikation ist dabei keineswegs einseitig geblieben, wenn auch aufgrund der wirtschaftlichen Entwicklung auf der einen und der Bevölkerungsentwicklung auf der anderen Seite die Gefälleprobleme immer krasser wurden. Mit der zunehmenden Ungleichheit der Austauschstrukturen wurden die bilateralen Beziehungen selbst in relativ nebensächlichen Punkten immer konfliktträchtiger. Das Bestehen der USA auf Einzelregelungen wurde für die Mexikaner immer nachteiliger, so daß sie sich seitdem bemühen, verschiedene Probleme zu einem "Paket" zusammenzuschnüren, um dadurch von den USA größere Zugeständnisse zu erreichen. Dieser Prozeß ist keineswegs abgeschlossen ${ }^{19}$, hat aber durchweg nicht die erhofften Ergebnisse gezeitigt. Zwar wurden die Beziehungen zwischen den USA und Mexiko bereits verschiedentlich als besonders reif und abgeklärt gewertet, allein aufgrund der Tatsache, daß man sich darüber verständigte, in einigen Fragen durchaus anderer Meinung $\mathrm{zu} \operatorname{sein}^{20}$, doch blieb es den Mexikanern nicht verborgen, daß eine solche Verständigung immer nur bei bestimmten Fragekomplexen möglich war.

Unter den drei typischen Konfliktformen, die entweder aufgrund von Nachbarschaftsproblemen oder von Nachbarschafts- und Gefälleproblemen oder aber nur von Gefälleproblemen zwischen Mexiko und den USA aufgetreten sind und zum Teil weiterhin eine erhebliche Rolle spielen, ist es die Mischung von Nachbarschafts- und Gefälleproblemen, die Mexiko die größten Schwierigkeiten macht. Hier ergeben sich nämlich Auswirkungen seiner internationalen Politik auf relativ begrenzte, meist ökonomische, bilaterale Probleme, die ihm nur die Option lassen, auf eine eigenständige Dritte-Welt-Politik zu verzichten oder aber massive Nachteile und wachsende Konflikte mit den USA in Kauf zu nehmen. Es erscheint deshalb nützlich, einmal die Fülle der Einzelkonflikte zwischen den beiden Staaten nach einem solchen Raster zu sortieren ${ }^{21}$.

\section{Nachbarschaftsprobleme}

Bei den eigentlichen Nachbarschaftsproblemen steht die genaue Regulierung der Grenze im Vordergrund: bei über $3000 \mathrm{~km}$ kein einfaches Problem. Die Gewässerund Nutzungsansprüche haben in den letzten Jahren zu einer ganzen Fülle von Konflikten geführt, die z. T. nur in sehr langwierigen Verhandlungen gelöst werden konnten ${ }^{22}$. Diese Probleme des eigentlichen Grenzverlaufs stellen sich mindestens dreifach: einmal handelt es sich um das $1600 \mathrm{qkm}$ große Terrain des Chamizal, das durch die ständigen Änderungen des Flußlaufes des Rio Grande einmal mexikanisches und dann wieder US-Gebiet geworden war, und in dem durch Besiedlung und Besitzansprüche beide Länder ihre Rechte geltend machten. Die Kennedy-Regierung hatte 1963 bereits eine „endgültige“ Regelung vereinbart ${ }^{23}$,

19 Bezeichnend dafür waren die Kommentare in der amerikanischen Presse, während des Portillo-Besuchs vom 13.-16. 2. 1977; siehe insbesondere "N. Y. T.“ vom 15. 2. 1977.

$20 \mathrm{Vgl}$. Cline, a. a. O., S. 388.

21 Für einen ähnlichen Katalog der Einzelkonflikte, siehe Brown/Wilkie: „Recent United States - Mexican Relations: Problems Old and New “ in: Twentieth Century American Foreign Policy, hrsg. von Braeman, John u. a., Ohio 1971, S. 378-419 und Brown, Lyle C.: "The Politics of United States Mexican Relations: Problems of the $1970 \mathrm{~s}$ in Historical Perspective “ in: Contemporary Mexico. Papers of the IV. International Congress of Mexican History, hrsg. von Wilkie, James W. u. a. Berkeley 1976, S. $471-493$

22 Vgl. Brown/Wilkie, a. a. O., S. 391-392.

23 einen guten Ưberblick über die vorausgegangenen Verhandlungen gibt US-Senate, Committee on Foreign Relations, Hearings: Convention with Mexico for Solution of the Problem of the Chamizal, Washington 1963. 
nach der das Chamizal-Gebiet an Mexiko zurückging, dafür aber Teile des Córdoba Island an die USA abgetreten wurden. Aber erst 1970 vereinbarten Mexiko und die USA, alle noch bestehenden territorialen Streitigkeiten zu beenden und den Rio Grande sowie den Colorado River in ihrem jetzigen Verlauf als nationale Grenze anzuerkennen ${ }^{24}$.

Abgesehen von diesen Problemen haben beide Grenzflüsse durch zunehmenden Salzgehalt immer wieder Konflikte zwischen den beiden Nachbarstaaten hervorgerufen. Im Rio Grande stieg seit 1962 durch den Bau des El Murillo-Kanals in Texas der Salzgehalt rasch an. Die daraufhin einsetzenden Ernteschäden in Mexiko, wo der Rio Grande als Hauptbewässerungsquelle für die Landwirtschaftsprojekte im Norden benutzt wird, führten zu langwierigen Auseinandersetzungen mit den USA. 1965 stimmten die Präsidenten Johnson und Díaz Ordáz schließlich dem Vorschlag einer internationalen Grenz- und Wasserkommission zu, das salzhaltige Wasser nicht mehr in den Rio Grande, sondern über einen neuen Kanal direkt in den Golf von Mexiko zu leiten. Die Kosten für dieses aufwendige Projekt übernahmen beide Staaten gemeinsam, und nachdem 1969 dieser Kanal fertiggestellt wurde, war das Salzproblem des Rio Grande weitgehend gelöst ${ }^{25}$.

Wesentlich schwieriger gestaltete sich eine Vereinbarung über die Entsalzung des Colorado River, der seit 1961 aufgrund von Bewässerungsmaßnahmen auf US-Seite und den damit verbundenen Kanalbauten eine ungewöhnlich hohe Steigerung seines Salzgehaltes zu verzeichnen hatte. Trotz des Baus eines Umgehungskanals, der bereits 1965 fertig wurde ${ }^{26}$, stieg der Salzgehalt weiterhin an. Nach einem Treffen der Präsidenten Díaz Ordáz und Nixon 1970 wurde ein weiterer Vertrag unterzeichnet ${ }^{27}$, der eine Verbesserung dieser Situation zwar in Aussicht stellte, aber schließlich nicht erbrachte. Erst 1973 wurde Mexiko eine bewässerungsgeeignete Wasserqualität des Colorado River zugesichert, die durch den Bau einer Entsalzungsanlage in den USA erreicht werden soll28.

Den Mexikanern ist es unverständlich geblieben, warum die USA nur mit Mühe zu verhältnismäßig geringen Zugeständnissen bei der Regulierung der Grenzflüsse und ihrer wirtschaftlichen Nutzung bewegt werden konnten. Es hat eigentlich immer persönliche Begegnungen der jeweiligen Präsidenten notwendig gemacht, um auch nur minimale Fortschritte bei diesen Grenzproblemen zu erzielen, obwohl den USA durchaus bekannt sein mußte, in wie starkem Maße die besonders armen Gebiete Nordmexikos auf die Bewässerung durch die Grenzflüsse angewiesen waren.

Ein zweites Nachbarschaftsproblem hat sich aufgrund der ständigen Zunahme des Drogenkonsums in den USA und der illegalen Drogeneinfuhr seit Ende der sechziger Jahre entwickelt. Dabei ist die 1969 als „operation intercept“ ins Leben gerufene Grenzkontrolle von mexikanischer Seite als besonders unangenehm empfunden worden. Die psychologisch ungeschickte Maßnahme zeigte keinerlei Erfolg und wurde im Oktober 1969 durch ein bilaterales Abkommen gegen Produktion und Vertrieb von Drogen zwischen beiden Ländern ergänzt. Seit 1970 werden darüber hinaus die Rauschgiftfelder unter Militäreinsatz gezielt mit chemischen Mitteln

\footnotetext{
24 Vgl. Schmitt, a. a. O., S. 202.

25 Vgl. Brown/Wilkie, a. a. O., S. 399-400.

26 Vgl. Brown/Wilkie, a. a. O., S. 397-398, sowie Brown, a. a. O., S. 476 und 492.

27 Vgl. Brown, a. a. O., S. 476-477.

28 Den besten Ưberblick über die endgültige Lösung und die erwarteten Auswirkungen geben Brownell, Herbert und Eaton, Samuel D.: "The Colorado River Salinity Problem with Mexico" in: American Journal of International Law, Vol. 69, No. 2 (Apr:1 1975), S. 255-271.
} 
reduziert, ohne daß sich aber in den letzten Jahren deutliche Erfolge eingestellt hätten ${ }^{29}$. Immer noch kommen zwischen 70 und 90 Prozent des Heroins, das in den USA verbraucht wird, über die mexikanische Grenze - z. T. unter starker Beteiligung der US-Mafia. Seit 1972 haben die USA der mexikanischen Seite technische Spezialausrüstungen im Wert von über 40 Mio. US-Dollar - vor allem in Form von Hubschraubern - zur Verfügung gestellt, um den Drogenverkehr besser unterbinden zu können. Zusätzlich haben sie mit einem Touristenboykott gedroht, falls es den Mexikanern nicht gelingen sollte, die Produktion und den Schmuggel von Heroin zu unterbinden ${ }^{30}$.

Eine zusätzliche Problematik ist dadurch entstanden, daß inzwischen rund 600 US-Bürger in mexikanischen Gefängnissen sitzen, weil die Mexikaner genau jene Methoden zur Verfolgung von Drogenschmuggel angewandt haben, die die USA von ihnen erwarten. Diese Rückwirkungen der mexikanischen Maßnahmen sind freilich bei der öffentlichen Meinung in den USA auf ein sehr negatives Echo gestoßen, und López Portillo hat bei seinem jüngsten Besuch bei Präsident Carter einen raschen Häftlingsaustausch mit den USA angekündigt ${ }^{31}$. Auch die Nachbarschaftsprobleme aufgrund des Drogenverkehrs zeigen deutlich, daß die USA zwar jederzeit bereit sind, Mittel zur Verfügung zu stellen, um den Mexikanern die Durchführung der von ihnen erwünschten Maßnahmen zu erleichtern, daß sie aber so gut wie nie in der Lage sind, diese Maßnahmen auf die Mentalität der Mexikaner und ihre spezifische Empfindlichkeit abzustellen, und deshalb sehr überrascht sind, wenn aus technischen Maßnahmen umfangreiche politische Konflikte entstehen, deren Folgen dann nur noch auf höchster Ebene korrigiert werden können.

Das dritte der Nachbarschaftsprobleme betrifft die Auseinandersetzung um die Hoheitsgewässer. Vor den Küsten Mexikos hatten sich amerikanische Fischfangflotten und mexikanische Fischer viele Jahre lang die ertragreichen Fanggründe streitig gemacht. $1967 \mathrm{kam}$ es zu einem Fischereiabkommen über 5 Jahre ${ }^{32}$. Nach dessen Ablauf hofften die USA, durch die Ergebnisse der UN-Seerechtskonferenz diese Problematik generell regeln zu können, ohne ein spezifisches Abkommen mit Mexiko treffen zu müssen. Präsident Echeverría dehnte jedoch bereits 1975 die wirtschaftliche Nutzzone Mexikos auf 200 Seemeilen aus, um die mexikanische Fischindustrie zu fördern, aber auch gleichzeitig eventuelle Ölfelder zu sichern $^{33}$. Diese Maßnahme rief den energischen Widerstand der USA hervor, die darin einen Versuch Mexikos sahen, durch die normative Kraft des Faktischen die Position der Dritten Welt beispielhaft durchzusetzen. Im August 1976 kam es schließlich dennoch zu einem bilateralen Abkommen zwischen Mexiko und den USA, das die Fischereirechte ab 1977 für die US-Fangflotten in der Wirtschaftszone Mexikos reduziert und Jahreslizenzen für die US-Fischer notwendig macht ${ }^{34}$.

Bei diesem Problem der Küstenfischerei beginnt das reine Nachbarschaftsproblem zumindest partiell auch ein Gefälleproblem zu werden, denn für die Mexikaner

$29 \mathrm{Vgl.} \mathrm{Brown,} \mathrm{a.} \mathrm{a.} \mathrm{O.,} \mathrm{S.} \mathrm{482-485.}$

30 Siehe Análisis Politico vom 9. 8. 1976.

31 Vgl. "NZZ ${ }^{\alpha}$ vom 18. 2. 1977.

32 Vgl. dazu Schmitt, a. a. O., S. 204-205 und Brown/Wilkie, a. a. O., S. 405-406.

33 Einen historischen Überblick über Mexikos Politik in der. Seerechtsfrage gibt: Vargas, Jorge E.: „Significado y trascendencia para México de un mar patrimonial de 200 millas; in: Comercio Exterior, "Vol. 25, No. 10 (Okt. 1975), S. 1146-1155, siehe zusätzlich: Scheffler, Gerhard: "Mexiko führt die ,Exklusive Wirtschaftszone“ vor seinen Küsten ein“ in: Verfassung und Recht in Úbersee, Vol. 9, No. 3, 1976, S. 373-376.

34 Vgl. Análisis Político vom 16. 8. 1976. 
sind die Nutzungsmöglichkeiten ihrer Küstengewässer wirtschaftlich bedeutsamer als für die Fischereiflotten der USA.

Obwohl die mexikanische Marine keineswegs in der Lage gewesen wäre, den Anspruch auf die 200-Meilen-Wirtschaftszone auch durchsetzen zu können, haben die USA sich offensichtlich im Hinblick auf nachbarschaftliche Gründe mit den Mexikanern in dieser Frage geeinigt. Wie schwer solche Einigungen jedoch erreichbar sind, wenn Nachbarschafts- und Gefälleprobleme zusammenfallen und in ihrem Konfliktgehalt kaum noch trennbar sind, mag die folgende Kategorie innerhalb des Konfliktrasters zwischen Mexiko und den USA verdeutlichen.

\section{Nachbarschafts- und Gefälleprobleme}

Bei den Nachbarschafts- und Gefälleproblemen handelt es sich um die weitaus größte Gruppe innerhalb der bilateralen Konflikte zwischen den beiden Staaten. Alle diese Probleme sind gleichermaßen davon geprägt, daß sie als reine Nachbarschaftsprobleme entweder gar nicht in Erscheinung träten, weil sie eben vornehmlich durch das Gefälle zwischen den USA und Mexiko zustandegekommen sind, oder aber doch nicht sonderlich konfliktträchtig wären. Dort, wo es sich eigentlich um reine Gefälleprobleme handeln würde, werden sie durch die Nachbarschaft beider Staaten verschärft bzw. in einen globalen Zusammenhang gestellt, der ein Arrangement zwischen Mexiko und den USA sehr erschwert.

Ein typischer Fall für diese Art Mischkonflikte ist der starke amerikanische Tourismus nach Mexiko. Auf diesem Gebiet hatte Mexiko gehofft, aufgrund seiner geographischen Vorteile und kulturhistorischen Schätze eine für seine Wirtschaftsentwicklung wichtige Industrie aufbauen zu können. Während der sechziger Jahre hat der Tourismus Mexikos Wirtschaft davor bewahrt ${ }^{35}$, aufgrund sinkender Exportpreise bereits damals in ähnliche Zahlungsbilanzschwierigkeiten zu geraten wie viele andere Länder Lateinamerikas ${ }^{36}$. Abgesehen von den strukturellen Fehlentwicklungen, die der Tourismus in Mexiko wie in anderen Entwicklungsländern mit sich gebracht hat, und $\mathrm{zu}$ denen auch die rasche Ubernahme der touristischen Infrastruktur durch nordamerikanische Unternehmen gezählt werden muß, ließ dieser Tourismus außerdem die mexikanische Wirtschaft gegenüber Pressionen aus den USA um so anfälliger werden. Der Boykott der jüdischen US-Touristen im Winter 1975/76, von dessen Folgen sich die mexikanische Tourismusindustrie immer noch nicht erholt hat ${ }^{37}$, ist nur ein Beispiel für die direkten Rückwirkungen mexikanischer Außenpolitik - wie in diesem Falle sein Abstimmungsverhalten in den UN - auf seine Beziehungen zu den USA ${ }^{38}$.

Einer der Hauptgründe für die Peso-Abwertungen von 1976 war die Sorge um den nachlassenden Tourismus aus den USA und das Bemühen, durch ein stärkeres Preisgefälle die verlorenen Touristenscharen wiederzugewinnen ${ }^{39}$. Ein Nebeneffekt der Tourismusproblematik ist die Auseinandersetzung um den Lufttransport zwi-

35 Der Tourismus erbrachte im letzten Jahrzehnt durchschnittlich 44 Prozent der jährlichen Deviseneinnahmen. „FAZ“ vom 12. 2. 1977.

$36 \mathrm{Vgl}$. Brody, a. a. O., S. 324.

37 Für 1975 wird der Rückgang der Tourismuseinnahmen mit 20 Prozent und für 1976 mit 14 Prozent angegeben. „FAZ“: Blick durch die Wirtschaft vom 12. 2. 1977.

$38 \mathrm{Zu}$ den Auswirkungen der mexikanischen Stimmabgabe gegen Israel in der Zionismusdebatte der UN, vgl. Ojeda, a. a. O., S. 197.

39 Siehe „Financial Times ${ }^{\alpha}$ vom 11. 2. 1977. 
schen beiden Ländern, bei der viele Jahre lang keine Einigung darüber erreicht werden konnte, in welchem Verhältnis US-Gesellschaften und mexikanische Luftlinien am Transport der Touristenströme beteiligt sein sollten ${ }^{40}$. Auch im Tourismus ist die extreme Ausrichtung nach dem Nachbarn im Norden dazu angetan, ständige Pressionsmöglichkeiten seitens der USA bei sogenanntem mangelndem Wohlverhalten ausgesetzt zu sein, zumal eine Diversifizierung der Touristenströme - etwa durch Europäer — nur bedingt möglich ist.

Noch krasser sind diese asymmetrischen Beziehungen im Hinblick auf den Handelsverkehr zwischen beiden Staaten. Während die USA als Weltwirtschaftsmacht trotz des relativ großen Handelsvolumens mit Mexiko von dem Warenaustausch mit Mexiko weitgehend unabhängig sind, schwanken die mexikanischen Importe und Exporte aus und nach den USA ständig zwischen 60 und 70 Prozent. Die steigenden Handelsdefizite auf mexikanischer Seite konnten dabei durch die Touristendollars in den letzten Jahren zunehmend weniger ausgeglichen werden. Der leichte Anstieg des Handels mit den LAFTA-Ländern, Japan und Westeuropa in jüngster Zeit hat nur wenig zur Verringerung dieser Abhängigkeit gegenüber den USA beitragen können ${ }^{41}$. Die schwankende US-Importpolitik ist sicherlich nicht der einzige Grund für die geringen Möglichkeiten Mexikos, seine Exporte auszuweiten. Sie trug aber dazu bei, daß die Handelsbilanzdefizite Mexikos sich zunehmend vergrößert haben ${ }^{42}$. Besonders der Schock von 1971, der durch einen 10\%igen Steueraufschlag der USA auf alle ihre Importe - unabhängig vom Herkunftsland - ausgelöst wurde, hat den Mexikanern gezeigt, wie stark ihre Abhängigkeit von den USA ist. Darüber hinaus haben sie erkennen müssen, daß die vielgerühmte "special relationship“ in so essentiellen Fragen wertlos für sie ist ${ }^{43}$. Der „Trade Act" von 1974 mit seinen Wohlverhaltensklauseln, die sich direkt gegen eine mögliche OPEC-Mitgliedschaft Mexikos richteten, hat die Abhängigkeit Mexikos zusätzlich verdeutlicht ${ }^{44}$. Echeverría hat sehr rasch eingesehen, daß bilaterale $\mathrm{Ab}$ machungen im Bereich des Außenhandels keinen großen Fortschritt für Mexiko mit sich bringen, und daß nur eine Diversifizierung der Außenhandelspartner langfristig die Abhängigkeit von den USA verringern könne. Seine eigene Politik hat allerdings gezeigt, daß die Hindernisse für einen solchen Prozeß aufgrund der geographischen Lage und der strukturellen Situation Mexikos kaum überschätzt werden können. Der einzige andere Ausweg, den Mexiko für diese Problematik sieht, ist eine globale Regelung im Rahmen einer neuen Weltwirtschaftsordnung, für die sich Echeverría besonders stark eingesetzt hat.

Ein weiteres typisches Problem, in dem sich Nachbarschaft und Gefälle mischen, ist die Grenzindustrie, die seit 1966 in einer 12 Meilen breiten Freihandelszone an der US-mexikanischen Grenze eingerichtet wurde. Mexiko hat ein Interesse daran, die besonders unterentwickelte nördliche Zone seines Staatsgebietes zu fördern und die in diesem Bereich extrem hohe Arbeitslosigkeit abzubauen. Von seiten der USA hat die Einrichtung von arbeitsintensiven Industriezweigen in diesem Grenzbereich

$40 \mathrm{Zu}$ den Einzelheiten vgl. Schmitt, a. a. O., S. 209-212.

41 Vgl. die Tabellen bei Schmitt, a. a. O., S. 257 und die Darstellung der Außenhandelsproblematik bei Ojeda, a. a. O., S. 140-152.

42 Das Außenhandelsdefizit gegenüber den USA betrug 1976 2,5 Milliarden US-Dollar. „N. Y. T. ${ }^{\star}$ vom 2. 2. 1977.

43 Siehe dazu Brody, a. a. O., S. 326, Anmerkung 40.

44 Für eine ausführliche Dokumentation über die Auswirkungen des Trade Act vom 20. 12 . 1974, vgl. "La Ley de Comercio de 1974 de Estados Unidos: Análisis y documentos" in: Comercio Exterior, Vol. 25, No. 5 (Mai 1975), Suplemento. 
den Zweck, die niedrigen Lohnkosten Mexikos auszunutzen, um dadurch Güter produzieren zu können, die mit den Importen aus industrialisierten Billiglohnländern konkurrenzfähig sind. Diese verlängerten Werkbänke waren Mexiko wirtschaftlich immerhin so von Nutzen, daß 1971 die Errichtung solcher Unternehmen, die ausschließlich für den Re-Export arbeiten, aufs ganze Staatsgebiet ausgedehnt werden konnte ${ }^{45}$.

Diese kurzfristigen Erfolge bei der Arbeitsplatzbeschaffung dürfen aber nicht darüber hinwegtäuschen, daß diese Grenzindustrien ganz erhebliche soziale Probleme aufwerfen. Zumeist werden nur Frauen eingestellt, für die außerdem keinerlei Arbeitsplatzsicherung besteht. Das Management und die Angestellten kommen ohnehin von der anderen Seite der Grenze. Bei Produktumstellungen kommt es häufig zu Massenentlassungen. Darüber hinaus wird aufgrund der mangelnden Integration dieser Industrien in die mexikanische Wirtschaft keinerlei Entwicklungseffekt erzielt. Außerdem werden die Grenzindustrien von den USGewerkschaften als Arbeitsplatzexporte scharf bekämpft und bleiben daher ein ständiger Streitpunkt in den Beziehungen zwischen Mexiko und den USA.

Eines der schwierigsten und nahezu unlösbaren Probleme, das am deutlichsten als Nachbarschafts- und Gefälleproblem ins Auge fällt, ist die Frage der mexikanischen Wanderarbeiter bzw. der illegalen Immigration in die USA. Obwohl genaue Zahlen selbstverständlich nicht bekannt sind, gibt es heute bereits Schätzungen, daß ca. 15 Millionen Mexikaner in den USA leben, die entweder bereits eingebürgert sind oder dort als sogenannte „residentes“ oder aber illegal im Lande leben. Die Zahl der Illegalen wird mit mindestens 6 Millionen $^{46}$ angegeben. Dazu kommen im Schnitt pro Jahr eine halbe Million hinzu. Dieses Problem ist freilich nicht nur für die USA, die zeitweilig diese Immigration gefördert haben, um billige Arbeitskräfte für ihre landwirtschaftlichen Industrien im Süden und Südwesten zu bekommen, sehr vielschichtig, sondern auch für Mexiko, da ein Großteil der Mexikaner sich relativ rasch in den USA akklimatisiert und dann als "chicano" eine eigene Identität entwickelt, die gelegentlich zu Spannungen gegenüber den Zuständen in der früheren Heimat führt ${ }^{47}$.

Zwischen 1930 und 1964 hatten die USA ein sogenanntes „bracero“-Programm ${ }^{48}$ eingerichtet, das den Status der Wanderarbeiter regelte. Die Kritik an der Diskriminierung der mexikanischen Arbeitskräfte trug ebenso wie die zunehmende Arbeitslosigkeit in den USA und die vehemente Gegnerschaft der US-Gewerkschaften dazu bei, daß das Programm 1964 eingestellt werden mußte ${ }^{49}$. Seit dieser Zeit ist die Zahl der illegalen Immigranten ganz erheblich nach oben geschnellt. Gleichzeitig

45 Zur Entwicklung der Grenzindustrie, vgl. Schmitt, a. a. O., S. 243-245 und vor allem die detaillierte Darstellung bei: Villalobos Calderón, Liborio: "La indústria maguiladora extranjera en México: mal necesario de una sociedad subdesarrollada" in: Relaciones Internacionales, Vol. 1, No. 1 (April/Junı 1973), S. 5-21.

46 Vgl. Economist vom 5. 2. 1977. Zur mexikanischen Sicht der „invasión silenciosa “ siehe Bustamante, Jorge A.: "El debate sobre la invasión silenciosa“, in: Foro Internacional, Vol. 17, Nr. 3 (Jan./März 1977), S. 403-417. Eine völlige Neuregelung der Immigrationsfrage - die vor allem die illegalen mexikanischen Einwanderer betrifft - hat Präsident Carter dem US-Kongreß am 4. 8. 77 vorgeschlagen. Demnach sollen alle illegalen Immigranten, die vor dem 1. 1. 70 in die USA gekommen sind, den Status von „permanent residents" erhalten. Alle vor dem 1. 1. 77 in die USA Gekommenen dürfen eine auf fünf Jahre befristete Aufenthaltsgenehmigung beantragen, und nur die seit diesem Datum illegal Eingewanderten sollen deportiert werden. Als flankierende Maßnahmen zur Reduzierung der illegalen Einwanderung sind verstärkte Kontrollen an der mexikanischen Grenze ebenso vorgesehen wie die Einführung von Bußgeldern in Höhe von $1000 \$$ für jeden eingestellten, illegal eingewanderten Arbeitnehmer bei den US-Arbeitgebern. Vgl. ,IHT" vom 6. 8. 77.

47 Für Einzelheiten siehe Brown, a. a. O., S. 488-490.

48 Vgl. Zea, Irene: "Los braceros: un problema humano“ in: Relaciones Internacionales, Vol. 2, No. 6

49 Dazu Brown/Wilkie, a. a. O., S. 387-388. 
haben die USA immer wieder versucht, durch Gesetzesvorlagen die Einstellung von illegalen Immigranten - die zumeist von den Unternehmern in skandalöser Weise ausgebeutet werden - zu unterbinden und die illegalen Immigranten nach Mexiko zurückzuschicken. Während sich letzteres durch verbesserte polizeiliche Kontrollen weitgehend durchsetzen ließ50, war eine Unterbindung der Einstellung in den USA bisher nicht zu erreichen. Die billige Arbeitskraft der "wetbacks“ - so der amerikanische Ausdruck für die illegalen Immigranten ${ }^{51}$ - fand vor allem im US-Senat allzu viele auf seiten der großen Wirtschaftsinteressen stehende Verteidiger. Präsident Carter hat angekündigt, daß er erneut einen Versuch unternehmen werde, eine gesetzliche Regelung für die Zahlung von Mindestlöhnen an die mexikanischen Arbeiter durchzusetzen. Dies würde automatisch zu einer wesentlich geringeren Einstellung von Mexikanern führen und somit den Anreiz für die illegale Immigration in die USA erheblich reduzieren. Damit wäre freilich für Mexiko, das einen Teil seiner Arbeitslosigkeit bisher in die USA exportieren konnte, das Problem keineswegs gelöst. Vielmehr müßte es damit rechnen, in den nächsten Jahren einige Millionen Arbeitslose zusätzlich zu verkraften. Die Vorschläge der USA, das Problem der Arbeitslosigkeit und der daraus resultierenden illegalen Immigration langfristig durch eine starke Begrenzung des Bevölkerungswachstums zu lösen, sind in Mexiko auf erhebliche Kritik gestoßen ${ }^{52}$.

Seitdem Präsident Ford die Einwanderungsquote für legale Immigration auf 20000 pro Jahr für Mexiko limitiert hat ${ }^{53}$, besteht zunächst die Gefahr, daß die illegale Einwanderung noch ansteigen wird, da in den letzten acht Jahren durchschnittlich 40000 Mexikaner legal in die USA eingewandert sind. Neben der ohnehin bereits bestehenden scharfen Grenzüberwachung haben die USA nur geringe Möglichkeiten, die illegale Einwanderung langfristig unterbinden zu können, solange das Gefälle zwischen den USA und Mexiko in dem jetzigen Ausmaß bestehenbleibt. Das große Interesse der USA an wirtschaftlicher Stabilität in Mexiko steht daher in direktem Zusammenhang mit ihren Befürchtungen, ein allzu großer Teil der sozialen Probleme Mexikos könnte über die gemeinsame Grenze schwappen.

Während in den letzten Jahren der Strom der Menschen über die Grenze nach Norden anstieg, verstärkte sich in der umgekehrten Richtung der Strom nordamerikanischen Privatkapitals nach Mexiko. Die Frage der Auslandsinvestitionen hat als geradezu historisches Thema vor und nach der Revolution die Beziehungen zwischen beiden Staaten wenn nicht konditioniert, so doch außerordentlich stark beeinflußt. Die große Anziehungskraft, die Mexiko für nordamerikanisches Kapital ausgeübt hat und noch ausübt, geht auf eine Reihe von Faktoren zurück, deren unterschiedliches Gewicht sich zwar im Laufe der Jahrzehnte verschoben hat, die aber insgesamt zu dem großen finanziellen Engagement der USA in der mexikanischen Wirtschaft beigetragen haben. Während ursprünglich die Ausbeutung von Rohstoffen im Vordergrund stand, kam später die Notwendigkeit hinzu, in Mexiko produzieren zu müssen, um den dortigen Markt wahrnehmen zu können, oder aber aufgrund von Billiglöhnen und Standortvorteilen den US-Markt von Mexiko aus kostengünstig beschicken zu können. Neben diesen rein ökonomischen Gründen

\footnotetext{
50 So wurden in den letzten Jahren durchschnittlich 750000-800 000 illegal eingereiste Mexikaner nach Mexiko zurückgeschickt; siehe Economist vom 10. 6. 1976.

51 Für eine gründliche Untersuchung siehe Samora, Julian: Los Mojados - the Wetback Story, Notre Dame 1971.

52 Vgl. Analísis Político vom 2. 2. 1976.

53 Siehe IHT vom 23. 10. 1976.
} 
war insbesondere die Stabilität und Kalkulierbarkeit des politischen Systems Mexikos einer der Hauptanreize für die Auslandsinvestoren ${ }^{54}$. Mexikos Position gegenüber den Auslandsinvestitionen hat sich seit 1940 relativ gradlinig entwickelt. Mexiko war zwar an solchen Investitionen, die ursprünglich fast ausschließlich aus den USA kamen und jetzt auch noch mindestens 80 Prozent der knapp 4 Milliarden Auslandsinvestitionen ausmachen ${ }^{55}$, sehr interessiert, aber von Anfang an darum bemüht, die Bedingungen für diese Investitionen selbst bestimmen zu können $^{56}$. Sein Verständnis der Regulierung der Auslandsinvestitionen ist direkt mit dem Selbstbestimmungsrecht der Nationen verbunden und fand seinen stärksten Niederschlag in der Erdölverstaatlichung von 193857. Die Mexikaner haben deswegen auch relativ wenig Verstänndis dafür, daß die USA immer wieder alle Maßnahmen, die den Interessen der Auslandsinvestoren zuwiderliefen, mit ideologischen Argumenten und politischen und wirtschaftlichen Sanktionen zu bekämpfen suchten.

Trotz der Beschränkungen und Kontrollen, die in den letzten Jahren in zunehmendem Maße für Auslandsinvestitionen eingeführt worden sind und die hier nicht ausführlich dargestellt werden sollen ${ }^{58}$, spielen die Auslandsinvestitionen weiterhin eine erhebliche Rolle, vor allem in den sogenannten Wachstumsbereichen wie Chemie, Maschinenbau, Lebensmittelverarbeitung und Transportmittel. Die mexikanische Regierung war insbesondere deshalb über den Einfluß des ausländischen Kapitals zunehmend besorgter geworden, weil es dem Auslandskapital häufig weniger um Neugründungen als um die Übernahme bereits bestehender mexikanischer Firmen ging. Es kam daher während der sechziger und siebziger Jahre zu einer ausgesprochenen Denationalisierung der mexikanischen Industrie ${ }^{59}$. Die mexikanischen Bemühungen in den siebziger Jahren um eine stärkere Kontrolle nicht nur über ausländische Kapitalbeteiligungen und Marktbeherrschung, sondern auch im Bereich des Technologietransfers ${ }^{60}$, haben zwar zu einer Verlangsamung des Denationalisierungsprozesses geführt, aber bisher kaum ausgereicht, die erwünschte private oder staatliche Kontrolle über die Entwicklung der nationalen Wirtschaft zu garantieren. Entgegen den Erwartungen der ausländischen Investoren haben sowohl das Auslandsinvestitionsgesetz als auch das Gesetz zum Technologietransfer die Rolle des Staates gegenüber den ausländischen Investoren gestärkt, ohne daß es deshalb zu einer Kapitalflucht in erheblichem Umfang gekommen wäre ${ }^{61}$. Dennoch ist $\mathrm{zu}$ erwarten, daß in dem Maße, wie Mexiko aufgrund seiner Wirtschaftsprobleme gezwungen ist, verstärkt durch staatliche Regulierung in den

\footnotetext{
54 Vgl. Hirsch-Weber, a. a. O., S. 71.

55 Siehe dazu vor allem die ausführliche Darstellung von Newfarmer, Richard S. und Müller, William F. in: US-Senate: Multinational Corporations in Brazil and Mexico: Structural Sources of Economic and Noneconomic Power. Report to the Subcommittee on Multinational Corporations of the Committee on Foreign Relations, Washington 1975.

56 Siehe dazu vor allem Sepúlveda Bernardo und Chumacero, Antonio: La inversión extranjera en México, 1973 und: Sepúlveda, Bernardo/Pellicier de Brody, Olga/Meyer, Lorenzo: Las empresas transnacionales en México, México 1974.

$57 \mathrm{Zu}$ einer Analyse der Auswirkungen der Erdölverstaatlichung auf die bilateralen Beziehungen siehe die ausgezeichnete Darstellung bei Meyer, Lorenzo: México y Estados Unidos en el conflicto petrolero (1917-1942), México 1968.

58 Zum Gesetzestext siehe Wedel, Henning von: „Neue Mexikanische Wirtschaftsgesetze“ in: Verfassung und Recht in Übersee, Vol. 7, No. 1 (1974), S. 59-77.

$59 \mathrm{Zu}$ diesem Schluß kommen auch Newfarmer und Müller in ihrer Studie für den US-Senat, a. a. O.; aus mexikanischer Sicht siehe Aguilera Gómez, Manuel: La desnacionalización de la economía mexicana, México 1975.

60 Zum Gesetzestext siehe Wedel, a. a. O. Eine besonders ausführliche Analyse findet sich bei Villar, Samuel: „El sistema mexicano de regulación de la inversión extranjera: Elementos y deficiencias generales in: Foro Internacional, Vol. 15, No. 3 (Jan./März 1975), S. 331-378; vgl. außerdem: Schmitt, a. a. O., S. 249 und Ojeda, a. a. O., S. 194.

61 Vgl. Schmitt, a. a. O., S. 252.
} 
Wirtschaftsprozeß einzugreifen, die Auseinandersetzung um die Rolle und den Einfluß der Auslandsinvestitionen in Mexiko noch heftigen Belastungsproben im Verhältnis zu den USA ausgesetzt sein dürfte.

Das Problem der Auslandsinvestitionen ist direkt mit dem übergeordneten Nachbarschafts- und Gefälleproblem des mexikanischen Entwicklungsmodells verbunden. Die heftige Reaktion der USA auf ihnen nicht genehme Entwicklungsmodelle in der Dritten Welt ist hinreichend bekannt; um so begrenzter ist der Spielraum für Veränderungen in einem Nachbarland wie Mexiko. Während der Wachstumsperiode der mexikanischen Wirtschaft nach dem Zweiten Weltkrieg wurden die abhängigen Beziehungen zwischen den USA und Mexiko nur partiell oder gar nicht in Frage gestellt. Aber auch während dieser Zeit war Mexiko sorgfältig darauf bedacht, sein Entwicklungsmodell zumindest in einigen Bereichen nicht dem USEinfluß auszusetzen. So hat sich Mexiko als einziges lateinamerikanisches Land bereits 1952 geweigert, an dem Programm des „US Mutual Security Act“ teilzunehmen und hat daher auch keine Militärhilfe von den USA erhalten ${ }^{62}$ und den USA seinerseits auch keinerlei Stützpunkte auf seinem Territorium eingeräumt $t^{63}$. Aufgrund dieser Entscheidung ist Mexiko die spezifische Entwicklung, die viele lateinamerikanische Militärs seit den fünfziger Jahren durchgemacht haben, erspart geblieben. Gerade aufgrund der mangelnden Unterstützung des Militärs durch die USA ist es Mexiko gelungen, sein Militär nicht zur entscheidenden politischen Kraft werden zu lassen.

Außerdem hat es Mexiko sorgfältig vermieden, an gewissen bilateralen Entwicklungshilfeprogrammen der USA teilzunehmen. Das lag vor allem daran, daß die wirtschaftlichen Zuwachsraten während der sechziger Jahre ohnehin so beachtlich waren, daß man damals von einem "milagro mexicano" sprach. So hat Mexiko auch als eines der wenigen Länder Lateinamerikas die Entsendung von Peace-CorpsFreiwilligen aus den USA abgelehnt ${ }^{64}$.

Seitdem das Ende des "milagro mexicano“ trotz der Erdölfunde unübersehbar geworden ist, ist die Diskussion in Mexiko über die Effektivität des bisherigen Entwicklungsmodells intensiviert worden. Die wirtschaftlich notwendige Steigerung der Exporte scheint aufgrund der US-Außenhandelspolitik nahezu aussichts$\operatorname{los}^{65}$. Und die von Echeverría angestrebte Außenhandelsdiversifizierung hat bisher nur sehr geringe und sehr langsam wirkende Alternativen ermöglicht. Der Abbau der "dependencia“ von den USA gehörte zweifelsohne zu den laut verkündeten Prioritäten der Präsidentschaft Echeverrías ${ }^{66}$. Andererseits hat seine Amtszeit aber auch gezeigt, daß diese Abhängigkeit im Laufe ihrer Entwicklung innerhalb Mexikos bestimmte wirschaftliche und politische Interessengruppen geschaffen hat, die durchaus in der Lage sind, den Abbau der Abhängigkeit von den USA innenpolitisch außerordentlich $\mathrm{zu}$ erschweren, wenn nicht gar $\mathrm{zu}$ vereiteln. Diese „linkage groups"67 sind vermutlich die sichersten Garanten dafür, daß eine ernsthafte Belastung der Beziehungen zu den USA zumindest auf wirtschaftlichem

62 Vgl. Weil, a. a. O., S. 40, sowie die Darstellung bei Ojeda, a. a. O., S. 55-67.

63 Die 1942 gegründete "Joint US-Mexican Defense Commission ${ }^{\alpha}$ besteht allerdings als Kontaktstelle zu den US-Militärs weiter.

64 Vgl. Schmitt, a. a. O., S. 255 und Ojeda, a. a. O., S. 75-76. Der neue Präsident López Portillo scheint sich jetzt allerdings aufgrund der schwierigen wirtschaftlichen Lage Mexikos auch vermehrt um direkte Entwicklungshilfe von den USA bemühen $\mathrm{zu}$ wollen. Vgl. seine Rede vor dem "National Press Club" in Washington am 15. 2. 1977 bzw. „NZZ“ vom 18. 2. 1977.

65 Siehe Brody, a. a. O., S. 332.

66 Vgl. Análisis político vom 18. 8. 1975.

67 Siehe auch Grabendorff, a. a. O. 
Gebiet auch in Zukunft vermieden werden dürfte ${ }^{68}$. Wie deutlich auch von den USA die Problematik des mexikanischen Entwicklungsmodells gesehen wird, haben die sehr unterschiedlichen Reaktionen auf das unter Echeverría erkennbar werdende Veränderungspotential von seiten der USA gezeigt ${ }^{69}$.

Andererseits könnte es für die USA durchaus gefährlich werden, wenn sie langfristig die sozioökonomischen Probleme Mexikos ignorieren und weiterhin einen wirtschaftlichen Satellitenstaat als Billiglohn-Land und Touristenparadies an ihrer südlichen Grenze erhalten wollten. Eine solche Politik muß die politische Stabilität Mexikos gefährden ${ }^{70}$. Unter der Carter-Administration sind daher zumindest in Ansätzen Überlegungen erkennbar, vorrangig zur Sicherung von Mexikos politischer Stabilität beizutragen, anstatt auf einem bestimmten Entwicklungsmodell zu beharren. Dieses Entwicklungsmodell bringt der US-Wirtschaft zwar bisher erhebliche Vorteile ein, ist aber augenscheinlich immer weniger in der Lage, die mexikanischen Entwicklungsprobleme zu überwinden. Die Mexikaner drängen daher auf einen größeren Toleranzspielraum bei den sicherlich langsamen, aber unausbleiblichen Veränderungen ihres Entwicklungsmodells.

Seit Jahrzehnten schon hat das Bemühen der USA, ein ihnen genehmes Entwicklungsmodell in Mexiko durchzusetzen und zu sichern, zu besonderer Empfindlichkeit gegenüber bestimmten außenpolitischen Aktionen Mexikos geführt. Dies war immer dann der Fall, wenn die wirtschaftlichen Interessen der USA direkt oder indirekt gefährdet erschienen oder wenn die allzu starke außenpolitische Identifikation Mexikos mit einem anderen Entwicklungsmodell die USA befürchten ließen, Mexiko könne „vom rechten Wege abkommen“. Letzteres wurde besonders im Falle der Beziehungen Mexikos zu Cuba deutlich, da Mexiko als einziges Mitglied der OAS ${ }^{71}$ niemals die Beziehungen zu Cuba abgebrochen hatte ${ }^{72}$. Die USA haben nie verstanden, daß Mexiko seine Politik vor allem unter dem Gesichtspunkt der Nichtinterventions-Doktrin gesehen hat.

Unabhängig von Cuba ist die Lateinamerikapolitik seit der Präsidentschaft von López Mateos und verstärkt während der Amtszeit Echeverrías zu einem Kernstück der mexikanischen Außenpolitik geworden. Insofern wirkt die Lateinamerika-Politik der USA daher direkt auf ihre bilateralen Beziehungen zu Mexiko zurück, so daß es den USA kaum gelingen dürfte, ihr Verhältnis zu Mexiko zu verbessern, wenn sie bestimmte lateinamerikanische Forderungen, zu deren Wortführer sich Mexiko gemacht hat, nicht berücksichtigen ${ }^{73}$. So hat die US-Politik gegenüber Allendes Chile - ähnlich wie zuvor im Falle Cubas - sicherlich die bilateralen Beziehungen zu Mexiko direkt und negativ beeinflußt ${ }^{74}$. In diesem

$68 \mathrm{Zu}$ diesem Schluß kommt auch Brody, a. a. O., S. 333.

69 Für eine generelle Einschätzung siehe Holt, Pat M., "The Political Aspects ${ }^{\star}$, in: US-Senate, Committee on Foreign Relations, Subcommittee on American Republics Affairs: Survey of the Alliance for Progress, Washington 1969, hier S. 19/20. Bei den jüngsten Reaktionen sind vor allem zwei aus dem letzten Jahr der Echeverría-Regierung erwähnenswert: 1. Ein Brief von 30 Abgeordneten an Präsident Ford, vom 5. 5. 76, in dem auf die kommunistische Entwicklung in Mexiko aufmerksam gemacht wird, vgl. 5. 5. 76, in dem auf die kommunistische Entwicklung in Mexiko aufmerksam gemacht wird, vgl.
Análisis Político vom 16. 8. 76. 2. Ein Brief des Direktors des "Committee for an Open Society* Análisis Político vom 16. 8. 76. 2. Ein Brief des Direktors des "Committee for an Open Society* lichen Probleme Mexikos nicht stärker berücksichtigt würden. Vgl. Análisis Político vom 15. 11. 76.

$70 \mathrm{Vgl}$. Economist vom 10.7. 76.

71 Auf Mexikos Politik in der OAS kann an dieser Stelle nicht näher eingegangen werden. Einen guten Úberblick gibt Pellicier de Brody, Olga: „México en la OEA“, in: Foro Internacional, Vol. 6, Nr. 2/3, (Okt./Dez., Jan./März 1965/66), S. 288-302."

72 Zur Cuba-Politik Mexikos, vgl. die vorzügliche Studie von Pellicier de Brody, Olga: México y la Revolución Cubana, México, 1972.

73 Vgl. Castañeda, Jorge: „Revolution and Foreign Policy: Mexico's Experience ${ }^{\alpha}$, in: Latin American International Politics, hrsg. von Astiz, Carlos Alberto, Notre-Dame 1969, S. 137-174, hier S. 161.

74 Vgl. Abriola, Carlos: „El Acercamiento mexicano - chileno“, in: Centro de Estudios Internacionales (Hrsg.) México y América Latina: La nueva política exterior, México 1974, S. 85-125. 
Zusammenhang wird offenbar, wie schwierig sich die Beziehungen zwischen den USA und Mexiko immer dort gestalten, wo sich bilaterale Probleme mit Problemen der Dritten Welt mischen, und wie empfindlich die USA immer dann reagieren, wenn Mexiko sich in den Auseinandersetzungen zwischen der Dritten Welt und den USA eindeutig mit der Dritten Welt identifiziert. Ein typisches Beispiel dafür war die von Mexiko betriebene Gründung der SELA, weil damit die Lateinamerikaner im Bewußtsein um die Notwendigkeit der "collective selfreliance" bewußt Front gegen die USA gemacht haben ${ }^{75}$. Auch als Mexiko 1976 ankündigte, es wolle der OPEC betreten, ließen die USA verlauten, daß sie dies einerseits als unfreundlichen Akt betrachten würden und andererseits Mexiko somit automatisch von den Vorteilen des "US Trade Act“ von 1974 ausgeschlossen würde. Mexiko hat darauf hin seine Absichtserklärung zwar zurückgezogen, aber sich bei seiner Preisgestaltung der Erdölexporte in die USA weitgehend der OPECPolitik angepaßt ${ }^{76}$.

In diesem Bereich der Nachbarschafts- und Gefälleprobleme sind mit Abstand die schwierigsten Belastungen der Beziehungen zwischen beiden Staaten aufgetreten. Es gibt keinerlei Anzeichen, daß sich das in Zukunft ändern wird; vielmehr muß sich Mexiko immer dann besonders getroffen fühlen, wenn es seine internationalen Aktionen und Interessenvertretungen in bilateralen Problemen von den USA bestraft sieht. Obwohl den Mexikanern ihr relativ geringer außenpolitischer Spielraum bewußt ist, reagieren sie auf diese politische Taktik des „Kolosses im Norden“ besonders empfindlich. Die Bilanz der Nachbarschafts- und Gefälleprobleme ergibt, daß die USA gerade in diesem Bereich große Vorteile aus der Abhängigkeit Mexikos ziehen. Die offiziellen Erklärungen über Belastungen und Mißverständnisse in den bilateralen Beziehungen sind daher oft von Empfindlichkeiten beider Seiten geprägt, die in einem ganz anderen politischen Bezug stehen als dort, wo sie ausgesprochen werden bzw. zur Anwendung kommen.

\section{Gefälleprobleme}

Innerhalb des Konfliktrasters zwischen Mexiko und den USA ist das dritte Problembündel - das der Gefälleprobleme - die kleinste Gruppe. Darunter lassen sich vor allem die Probleme der Kredite und die Dritte-Welt-Politik subsumieren, wobei die "Charta der wirtschaftlichen Rechte und Pflichten der Staaten 77" der deutlichste Ausdruck für die Dritte-Welt-Politik Mexikos ist. In diesem Zusammenhang muß auch Mexikos Verhalten in den UN gesehen werden, dessen Spezifika aber hier ausgeklammert bleiben sollen ${ }^{78}$. Sowohl bei den Krediten als auch bei der Dritte-Welt-Politik ist die Grenze fließend zu den Problemen, die als Nachbarschafts- und Gefälleprobleme bezeichnet werden können. Dennoch dürfte dabei das Nachbarschaftsverhältnis keine so wesentliche Rolle spielen wie bei vorher angesprochenen Problemkreisen.

\footnotetext{
75 Zum Stellenwert von SELA innerhalb der mexikanischen Außenpolitik, vgl. Ojeda, a. a. O., S. 191. Generell zu diesem. Thema: Javier Alejo, Francisco und Hurtado, Hector: El SELA. Un mecanismo para la acción, México 1976.

76 Siehe "Financial Times“ vom 25. 1. 1977

77 Zum Text der Charta siehe BMZ: Entwicklungspolitik, Materialien, Juni 1975, S. 11-21.

78 Zur Politik Mexikos in der UNO vgl. das Standardwerk von Robles, García Alfonso: México en las Naciones Unidas, 2 Vol. México 1970 und Faust, John R./Stansifer, Charles L.: „Mexican Foreign Policy in the UN: The Advocacy of Moderation in an Era of Revolution “, in: Astiz, Carlos A. (Hrsg.), a. a. O., S. $100-110$.
} 
Mexiko gehört zu den am höchsten verschuldeten lateinamerikanischen Ländern ${ }^{79}$, aber auch zu den Ländern mit einem besonders guten „credit rating“. Mexiko hat bei den zunehmenden Kreditaufnahmen in den letzten Jahren immer wieder versucht, diese Kredite weniger durch bilaterale Vereinbarungen oder private Banken zu bekommen, als vielmehr von internationalen Finanzinstitutionen, um dadurch direkte Pressionen $\mathrm{zu}$ vermeiden ${ }^{80}$. Mit den wachsenden wirtschaftlichen Schwierigkeiten seit 1975/76 ist auch der IMF verstärkt der wirtschaftlichen Stabilisierung Mexikos zu Hilfe gekommen und hat im Dezember 1976 einen Kredit von 1,2 Milliarden Dollar bereitgestellt, um für eine Periode von drei Jahren die Handels- und Zahlungsbilanzdefizite in Mexiko abbauen zu helfen ${ }^{81}$. Erfahrungsgemäß haben jedoch die IMF-Auflagen für die Wirtschaftspolitik des betroffenen Landes starke negative Auswirkungen auf die lohnabhängige Bevölkerung, so daß vielleicht gerade aufgrund der wirtschaftspolitischen Stützung durch den IMF die politische Stabilität durch soziale Proteste gefährdet werden könnte.

Das Profil Mexikos als Land der Dritten Welt ist erst in der Regierungszeit von Echeverría deutlich zum Ausdruck gekommen. Er hat es verstanden, die innere System- und Legitimationskrise durch die außenpolitische Hinwendung zur Dritten Welt zu mildern ${ }^{82}$ und damit gleichzeitig die „bargaining power" Mexikos gegenüber den USA zu erhöhen ${ }^{83}$. Der Höhepunkt dieser Politik war die von ihm vorgeschlagene "Charta der wirtschaftlichen Rechte und Pflichten der Staaten“, die er erstmals vor der UNCTAD III in Santiago de Chile 1972 postulierte. Sie wurde jedoch erst nach fast drei Jahren heftiger Kämpfe auf verschiedenen Ebenen, am 12. 12. 1974 von der UN-Vollversammlung gegen die Stimmen der USA und einiger Industriestaaten ${ }^{84}$ angenommen. Mit diesem Manifest der wirtschaftlichen Forderungen der Dritten Welt gegenüber den Industrieländern hat Mexiko seine Beziehungen $\mathrm{zu}$ den USA außerordentlich stark belastet. Insbesondere folgende Vorstellungen der Charta sind auf den Widerstand der USA gestoßen:

1) Das unumstößliche Recht der Staaten zur Nutzung ihrer eigenen Ressourcen;

2) das Recht der Staaten, die Verantwortlichkeit des Privatbesitzes gegenüber dem öffentlichen Interesse - also die Sozialbindung des Eigentums - zu definieren;

3) die Unterwerfung des Auslandskapitals unter die Gerichtsbarkeit des Gastlandes;

4) das Verbot der Einmischung in interne Angelegenheiten der Staaten durch multinationale Unternehmen;

5) die Abschaffung von Handelsdiskriminierungen und Wirtschaftssanktionen gegenüber den Entwicklungsländern;

6) die Notwendigkeit einer Preisstabilisierung für Rohstoffe;

\footnotetext{
79 Mexiko zahlt ca. drei Milliarden US-Dollar pro Jahr an die USA für Kreditrückzahlungen, Zinsen und Patentrechte, siehe Excelsior vom 10. 2. 77. Für eine umfassende Analyse des Verschuldungsproblems, vgl. Green, Maria del Rosario: „Deuda pública externa y dependencia. El caso de México ${ }^{\circ}$ in: La política exterior de México: Realidad y perspectivas, hrsg. vom Centro de Estudios Internacionales, México, 1972, S. 55-77.

$80 \mathrm{Vgl}$. Weil, a. a. O., S. 270. Inzwischen dürften allerdings mehr als die Hälfte der mit ca. 30 Milliarden US-Dollar angegebenen Auslandsschulden von Privatbanken gehalten werden. Vgl. Latin America: Economic Report, vom 18. 2. 77.

81 Siehe „N. Y. T. ${ }^{\alpha}$ vom 1. 1. 1977.

82 Vgl. Grabendorff, a. a. O.

$83 \mathrm{Zu}$ den Grenzen einer solchen Politik siehe Pellicier de Brody, Olga: „Tercermundismo del capitalismo mexicano: ideología y realidad ${ }^{\alpha}$, in: Cuardernos Políticos (Jan./März 1975), Nr. 3, S. 52-59.

84 Das Stimmenverhältnis in der Vollversammlung der UN war 120:6 bei 10 Enthaltungen. Dagegen stimmten außer den USA noch Belgien, Dänemark, BRD, Luxemburg und Großbritannien.
} 
7) die Herabsetzung der Kosten für den Technologietransfer;

8) die Forderung nach zinsgünstigen und nicht liefergebundenen Krediten der Industrieländer an die Entwicklungsländer ${ }^{85}$.

Diese Forderungen der Charta beinhalteten für Mexiko die Ausrichtung auf ein gänzlich anderes Entwicklungsmodell, als es die USA in ihrer unmittelbaren Nachbarschaft verwirklicht sehen wollen und spielten somit wiederum in beide Problembereiche, nämlich die Nachbarschafts- wie die Gefälleprobleme hinein. Trotz seiner Rolle als Sprecher der Dritten Welt ist ausgerechnet Mexiko nicht an den Nord-Süd-Verhandlungen in Paris beteiligt worden. Der Grund dafür dürfte eindeutig in den Interessen der USA und der Abhängigkeit Mexikos zu suchen sein ${ }^{86}$.

In den letzten Jahren ist das Bewußtsein der Mexikaner um Unterentwicklung und Abhängigkeit außerordentlich geschärft worden, was sicherlich nicht nur auf die verstärkte Identifikation Mexikos mit der Dritten Welt zurückzuführen ist, sondern auch die Krise des politischen Systems Mexikos - die seit 1968 evident geworden ist - und die verschlechterte wirtschaftliche Situation widerspiegelt. Auch dies ist ein Phänomen, das die USA nur sehr langsam zu begreifen scheinen und das sie durch die Konzeption der "special relationship“ lange Zeit zu überdecken suchten. Inzwischen ist ihnen die Nachbarschaft zu Mexiko als einem Land der Dritten Welt bewußter geworden, und sie wissen, daß Mexiko einen Teil seiner Probleme wie Bevölkerungsdruck, Arbeitslosigkeit, Elend und Kriminalität in die USA exportieren kann. Diese Entwicklung wird um so stärker, je weniger die USA bereit sind, durch Abbau von Handelshemmnissen und andere Maßnahmen bei einer Lösung dieser Probleme mitzuhelfen. Mexiko ist sich bewußt, daß es aufgrund der verschiedenen Nachbarschafts- und Gefälleprobleme - im Gegensatz zu anderen Ländern der Dritten Welt - die USA durch die gemeinsame Grenze zu Reaktionen auf diese Probleme zwingen kann ${ }^{87}$.

\section{Methoden und Instrumente der mexikanischen Außenpolitik}

Die Aussichtslosigkeit, sich gegen die USA durchsetzen zu können, hat angesichts der eher zu- als abnehmenden Abhängigkeit von den USA zu einer Doppelstrategie geführt: ein eher skeptisches Defensiv-Verhalten auf der einen Seite und die Ausnützung von Kooperationsmöglichkeiten mit anderen Staaten der Dritten Welt zur Durchsetzung der eigenen politischen Vorstellungen auf der anderen Seite. Es ist dieses Defensiv-Verhalten, das die klassische mexikanische Diplomatie auszeichnet und das auf der Verbindung von Völkerrechtsprinzipien mit einem gewissen moralischen Anspruch in der internationalen Politik beruht. So sind es auch legale und moralische Instrumente der Außenpolitik, die die entscheidende Rolle bei den politischen Aktionen Mexikos gespielt haben, weil sich Mexiko als ein Land versteht, das von eigentlicher Machtpolitk und der damit verbundenen Drohkapazität bei der Durchsetzung eigener Ansprüche ausgeschlossen ist ${ }^{88}$.

\footnotetext{
$85 \mathrm{Zu}$ den Zielvorstellungen der Charta vgl. vor allem Green, Maria del Rosario: „El nuevo orden económico internacional“, in: Foro Internacional, Vol. 15, Nr. 4 (April/Juni 1975), S. 493-534. 86 Vgl. Washington Post vom 5. 10. 76

87 Vgl. dazu den sehr aufschlußreichen Kommentar der „N. Y. T.“ vom 15. 2. 77.

88 Trotz dieser Selbsteinschätzung sehen die kleinen Staaten Zentralamerikas die Rolle Mexikos durchaus anders. Siehe dazu: Salisbury, Richard V.: ,The other Colossus: An Isthumian View of Mexico;, in: Latin American Studies Conference Proceedings, hrsg. vom Council on International Studies, Special Studies Nr. 42 (II), Buffalo 1973, S. 279-292.
} 
Die klassischen lateinamerikanischen Souveränitätsdoktrinen von Calvo und Drago $^{89}$ wurden durch die Estrada-Doktrin Mexikos ergänzt ${ }^{90}$. Diese Doktrin vom 27. 9. 1930 bildete die Grundlage für die extensiv interpretierte Nichtinterventions-Politik der mexikanischen Regierung. Dieses Prinzip der Nichtintervention ist deswegen zum Pfeiler der mexikanischen Außenpolitik geworden, weil es den negativen Erfahrungen Mexikos mit seinen Beziehungen zu den USA entstammt und der Begriff der Intervention nicht nur für die US-Beziehungen zu Mexiko, sondern auch zu Lateinamerika überhaupt kennzeichnend ist ${ }^{91}$. Die strikte Verteidigung der Nichteinmischung und der Selbstbestimmung geht vor allem auf die mexikanischen Erfahrungen während und nach der Revolution zurück ${ }^{\mathbf{9}}$. Mexikos Notwendigkeit, sein Territorium, seine Souveränität und seine Integrität zu verteidigen - wie es der ehemalige Präsident López Mateos ausdrückte ${ }^{93}$, hat seine Methoden und seine Instrumente im außenpolitischen Verhalten geprägt. Seine besondere Empfindlichkeit gegenüber dem Paternalismus der USA hat darin seinen Ursprung.

Gerade weil Mexiko den Nord-Süd-Konflikt so substantiell und paradigmatisch vorgelebt hat und deshalb zahlreiche Erfahrungen im Umgang mit der Führungsmacht der Industrieländer sammeln konnte, hat sein Gewicht in der Dritten Welt in den letzten Jahren so stark zugenommen. Die Erwartung der USA, durch bilaterale Abkommen Mexiko stärker an sich binden zu können, verkennt die Notwendigkeit für Mexiko, in einer "Allianz der Armen“ langfristig größere Zugeständnisse von den USA auszuhandeln. Insofern ist die verstärkte Identifikation mit der Dritten Welt nur eine logische Fortschreibung der moralischen und legalistischen Position der klassischen mexikanischen Außenpolitik. Denn der Mangel an "countervailing power" zwingt Mexiko geradezu, nach allgemeinen Prinzipien zu suchen, die seinen eigenen Forderungen gegenüber den USA größeren Nachdruck verleihen. Dazu hat während der Amtszeit Echeverrías sowohl die verstärkte Integration Mexikos in die Staatengemeinschaft Lateinamerikas ${ }^{94}$ als auch sein Image als einer der Wortführer der Interessen der Dritten Welt beigetragen. Es erscheint durchaus möglich, daß die Politik des neuen Präsidenten López Portillo ein erneutes Beispiel für die vieldiskutierte Pendeltheorie des mexikanischen Systems - in diesem Falle auf die Außenpolitik angewandt - abgibt ${ }^{95}$. So wie López Mateos für Mexiko das Fenster zur Welt aufgestoßen hatte und sich dann Díaz Ordáz wieder einzig dem Dialog mit den USA zugewandt hatte, so spricht einiges für die Wiederholung dieses Verhaltensmusters in bezug auf Echeverría und López Portillo96. López Portillo bemüht sich offensichtlich darum, eine neue Art „special relationship“97 mit den USA zu erreichen — nicht

$89 \mathrm{Vgl}$. dazu die vorzügliche Darstellung bei Krakau, Knud: „Lateinamerikanische Doktrinen zur Realisierung staatlicher Unabhängigkeit und Integrität", in: Verfassung und Recht in Ưbersee, Vol. 8, No. 2 (1975), S. $117-144$.

$90 \mathrm{Zu}$ den Möglichkeiten und Grenzen der Estrada-Doktrin vgl. Engel, James F.: "The Revolution and Mexican Foreign Policy“, in: Journal of Inter-American Studies, Vol. 11, No. 4" (Okt. 1969), S. 529, außerdem Schmitt, a. a. O., S. 265.

$91 \mathrm{Vgl}$. dazu auch die detaillierte Studie zum US-Engagement in Lateinamerika von Kane, William E.: Civil Strife in Latin America. A legal History of US-Involvement, Baltimore 1972, S. 1.

92 Vgl. dazu Castañeda, a. a. O., S. 137 und Engel, a. a. O.: „Mexican foreign policy, it may be simply stated, is an extension into international affairs of principles and practices developed and tested in the Mexican Revolution “, ebd., S. 532.

93 Vgl. Engel, a. a. O., S. 524 .

94 Vgl. Ojeda, a. a. O., S. 42.

95 Vgl. Kaye, Harvey F.: "How ,New" is Mexicos Foreign Policy?" in: Inter-American Economic Affairs, Vol. 28 , Nr. 4 (Frühjahr "1975), S. 87-92, hier S. 91.

96 Die typische Erwartungshaltung der USA läßt sich deutlich an einer Überschrift der „N. Y. T.“, vom 9. 9. 76 ablesen: "Mexico's Change of Presidents expected to improve US-ties.“

$97 \mathrm{Vgl}$. Christian Science Monitor, vom 30. 8. 76. 
nur aus wirtschaftlichen Gründen, sondern auch, weil er es mit Rücksicht auf die innenpolitische Machtverteilung in Mexiko für notwendig hält, die starke Orientierung Echeverrías zur Dritten Welt hin zu korrigieren.

Weil die Grenze zwischen den USA und Mexiko nicht nur eine Kulturschwelle ist, sondern auch Erste und Dritte Welt aufs deutlichste trennt, bleibt den Mexikanern keine andere Wahl, als all jene Konflikte exemplarisch durchzustehen, die weit weniger Nachbarschaftskonflikte als Gefälleprobleme zwischen Erster und Dritter Welt sind. Dabei handelt es sich nicht um isolierte Einzelprobleme, sondern fast ausschließlich um komplexe Strukturprobleme. Solange keine globalen Lösungen zur langsamen Überwindung bzw. wenigstens Verringerung des Gefälles zwischen Erster und Dritter Welt in Sicht sind, die auch von den USA akzeptiert werden, solange werden die Konflikte zwischen beiden Ländern eher zu- als abnehmen. 
serves to "settle" most of the disputes that arise among kinsmen and neighbors, but it also serves to educate citizens about current community standards. This paper describes the two features which appear to be most important in accounting for the popularity of the town hall court: (1) the ideology behind court procedures which stresses the intrinsic worth of each individual, thus requiring the reintegration of wrongdoers, and (2) the political system which rewards the powerful for heeding the wishes of the powerless.

\section{Overwhelming neighborhood: Mexico's relations with the United States}

\section{By Wolf GrabendorfF}

The neighborhood between the USA, a world power, and Mexico, a poor country, is of a special kind, since it has very little in common with the type of neighborhood the USA shares with Canada at its northern frontier. In contrast, the USA borders the Third World at its southern frontier. The Rio Grande is much more a cultural threshold than an ordinary border. The problems and conflicts which arise from this type of neighborhood, therefore, are much less "neighborhood" problems than "gap" problems. They are especially difficult to solve when the "neighborhood" problems also become "gap" problems. Therefore, a special type of relationship has developed throughout history between Mexico and the USA, whereby Mexico was forced to develop specific methods and instruments in order to face its overwhelming neighbor.

This paper deals with the three different types of conflicts which exist between these two structurally so very different countries. It shows that the specific "neighborhood" problems such as drug smuggling, fishing rights, and border regulations are probably the easiest to solve. Greater difficulties arise from the mixture of "neighborhood" and "gap" problems which account by far for the largest group. Among them are tourism, trade, border industries, illegal immigration, foreign investments, and the general implications of the Mexican development model. In all of these areas, the USA profits a great deal from Mexican dependency, and it is easily demonstrated that this type of bilateral conflict is much deeper rooted than it appears to be. The final and smallest conflict group consists of specific "gap" problems such as credits and Third Worldism as a foreign policy posture.

To cope with all these bilateral problems with the USA, the Mexicans have developed a double strategy: A more defensive legalistic approach on the one hand and the use of bargaining power through cooperation with the Third World on the other. The lack of countervailing power impels Mexico to seek general principles in international affairs where its national interests can be sustained more forcefully against the "colossus of the north". 